\title{
EDITORIAL
}

\section{Cryptosporidiosis en personas infectadas por el VIH en Venezuela: Potencial impacto de la crisis en el pais.}

Cryptosporidium es un problema de salud pública por ser uno de los protozoos patógenos más importantes como causantes de epidemias transmitidas por el agua y por su alto potencial oportunista, afectando en especial a individuos inmunocomprometidos. La criptosporidiosis se considera una de las infecciones oportunistas más riesgoosas para los pacientes con SIDA. Los rasgos clínicos de la infección varían en severidad dependiendo de la edad, el estado nutricional y el estado inmune del paciente (1), así como de las especies y genotipos de Criptosporidium (2). Los pacientes con VIH/ SIDA con recuentos de linfocitos CD4+ por encima de 180-200 células/ $\mu \mathrm{L}$ pueden ser asintomáticos o desarrollar una enfermedad diarreica auto-limitante. Sin embargo, la diarrea puede persistir durante varios meses en pacientes con recuentos de linfocitos CD4 $+<50-100$ células $/ \mu \mathrm{L}$, lo cual resulta en deshidratación severa, pérdida de peso, desnutrición e incluso la muerte. Estos pacientes están en mayor riesgo de desarrollar infecciones extraintestinales (3). El diaǵnóstico inicial de criptosporidiosis en pacientes con SIDA es un factor de mal pronóstico. Su prevención es especialmente importante en estos pacientes por el gran riesgo de desarrollar una enfermedad severa y fulminante.

Aún no existen drogas o vacunas efectivas para el tratamiento o prevención de la criptosporidiosis en pacientes infectados por el VIH. El tratamiento antirretroviral (TAR) tiene un efecto dramático sobre la infección en estos pacientes ya que conduce a la reducción de la carǵa viral y la recuperación de la cuenta de linfocitos $\mathrm{CD} 4+$, permitiendo la recuperación clínica o la erradicación del parásito. Hasta el presente, ésta es la terapia de elección en pacientes inmunocomprometidos y puede ser usada también profilácticamente (1). Sin embargo, este tratamiento es muy costoso y no está ampliamente disponible en muchos países en desarrollo. Debido a la falta de terapias efectivas, Cryptosporidium sigue siendo una gran amenaza para las personas con el VIH y la carǵa de la infección continúa alta en los países en desarrollo, ya que estos individuos tienen acceso limitado al TAR y la prevalencia de Cryptosporidium es significativamente mayor (4).

En un análisis de 270 estudios de 59 países, la prevalencia promedio de Cryptosporidium en pacientes con VIH/SIDA fue de $8,6 \%$ y de $13,1 \%$ en pacientes con el VIH, significativamente mayor a $5,9 \%$ en individuos VIH negativos. La prevalencia promedio de criptosporidiosis en pacientes con VIH/SIDA en países industrializados y en desarrollo fue de $5,6 \%$ y 11,6\%, respectivamente. Los pacientes con VIH/SIDA y diarrea, los que tenían bajos recuentos de linfocitos CD4+ y aquéllos sin TAR tuvieron una prevalencia significativamente mayor de Cryptosporidium que otros pacientes (5).

Un meta-análisis de 161 estudios, publicados entre 2000 y 2017, sobre las infecciones por Cryptosporidium en pacientes con VIH muestra que la prevalencia mundial del parásito en estos pacientes es de 14,4\%. Con un número global de 36 millones de pacientes infectados por el VIH, se estima que 4 millones de éstos están coinfectados por 
Cryptosporidium. El tener diarrea y $<200$ células $\mathrm{CD} 4 / \mu \mathrm{L}$ aumenta el riesgo de estos pacientes a la criptosporidosis, mientras que el uso de terapia antirretroviral disminuye significativamente el riesgoo a la infección (6).

En Venezuela, la criptosporidiosis es endémica y en pacientes con SIDA y diarrea se han observado tasas altas de infección de hasta 41,3\% (1). Nuestros estudios de parasitosis intestinales en el país durante décadas, incluyendo los patógenos emergentes Cryptosporidium y Cyclospora cayetanensis, revelan la permanencia de tasas altas de infección con una o más especies parasitarias y helmintos en áreas de bajos recursos económicos (1,7-9). Los helmintos tienen efectos inmunomoduladores sistémicos a través de la secreción de proteínas y por alteración del microbioma intestinal (10), pudiendo influenciar la severidad de otras infecciones. Esto constituye otra potencial amenaza para los pacientes con el VIH y Cryptosporidium, ya que este protozoo por si solo es un grave riesgo de enfermedad y muerte.

La acentuada crisis económica, política y social de Venezuela en las dos últimas décadas ha suscitado una erisis humanitaria sin antecedentes que se agrava cada vez más. El colapso del sistema de salud y de los servicios públicos ha determinado la reemergencia de enfermedades infecciosas ya controladas y diversas epidemias. La catastrófica situación del país afecta en especial a las personas infectadas por el VIH ya que el acceso al TAR es restringido y éste es necesario para evitar el SIDA, la transmisibilidad del virus y disminuir o evitar las coinfecciones y morbimortalidades asociadas. Por otro lado, en los laboratorios clínicos, no hay disponibilidad de métodos adecuados para la detección de Cryptosporidium y la única opción es el frotis fecal directo que tiene poca sensibilidad para el diagnnóstico del parásito.
La carencia de una infraestructura sanitaria apropiada, de saneamiento ambiental y de un nivel básico de agua potable asociada a una hiperinflación galopante, que implica un incremento mantenido de la pobreza y desnutrición, auspician la prevalencia, transmisión y epidemiología dinámica de los agentes infecciosos haciendo a estos pacientes más vulnerables a otras infecciones. Tal situación combinada al alto potencial oportunista de Cryptosporidium, la cronicidad y gravedad eventual de la infección señalan el gran riesgo de estos pacientes, muchos de los cuales migran en busca de tratamiento y una mejor calidad de vida al igual que millones de venezolanos han emigrado. Este éxodo hacia múltiples naciones producirá una gran conmoción global en la dinámica de transferencia del VIH, Cryptosporidium y otros agentes infecciosos.

La situación de las personas con VIH/ SIDA en Venezuela en medio de esta grave crisis es trágica. Las perspectivas para estos pacientes son desesperanzadoras ya que ante esta realidad solo se puede vislumbrar una mala calidad de vida y una corta sobrevivencia. En este país, se ha abierto una caja de Pandora y se han desatado todos los males y la esperanza, que es lo único que queda, no se vislumbra a corto plazo. Se requiere una ayuda humanitaria internacional con suma urgencia para aliviar el hambre, la morbilidad y la mortalidad de estos pacientes y de la mayoría de la población.

Leonor Chacín-Bonilla 


\section{Cryptosporidiosis in HIV infected persons in Venezuela: Potential impact of the country crisis.}

The high opportunistic and severe life-threatening potential of Cryptosporidium in HIV infected patients is a global public health problem. Persons with AIDS are susceptible to a devastating form of cryptosporidiosis. No effective drug therapy or vaccine are yet available for the infection in these patients. The best approach to prevention and therapy of cryptosporidiosis in them is the maintenance of the immune system function by using ART that allows clinical recovery or parasite eradication. As a consequence of the crisis, Venezuela is facing a collapse of the health system and public services with the worse sanitary conditions and limited water supply enabling the spread and outbreaks of infectious diseases. This situation along with the limited availability of ART, and high levels of poverty and malnutrition warranty an increase in the prevalence and morbimortality of eryptosporidiosis in HIV infected patients.

\section{REFERENCIAS}

1. Chacín-Bonilla L. Criptosporidiosis en humanos. Invest Clín 1995; 36: 207-250.

2. Chacín-Bonilla L. Importancia de las diferentes especies y genotipos en salud pública. Invest Clin 2001; 42: 83-85.

3. O'Connor RM, Shaffie R, Kang G, Ward HD. Cryptosporidiosis in patients with HIV/AIDS. AIDS 2011; 25: 549-560.

4. Iqbal A, Lim YAL, Mahdy MAK, Dixon BR, Surin J. Epidemiology of cryptosporidiosis in HIV infected individuals: A global perspective. Scientific Reports 2012; 1:431. https:// dx.doi:10.4172/scientificreports. 431.

5. Wang R, Li J, Chen Y, Zhang L, Xiao L. Widespread occurrence of Cryptosporidium infections in patients with HIV/AIDS: Epidemiology, clinical feature, diagnosis, and therapy. Acta Trop 2018; 187: 257-263.

6. Ahmadpour E, Safarpour H, Xiao L, Zarean M, Hatam-Nahavandi K, Barac A, Picot S, Rahimi MT, Salvatore Rubino S, Mahami-Oskouei M, Spotin A, Nami S, Hossein Bannazadeh Baghi HB. Cryptosporidiosis in HIV-positive patients and related risk factors: A systematic review and meta-analysis. Parasite 2020;27: 27. https://doi.org/10.1051/parasite/2020025

7. Chacín-Bonilla L, Dikdan Y. Prevalencia de Entamoeba histolytica y otros parásitos intestinales en una comunidad suburbana de Maracaibo. Invest Clin 1981; 22: 185-203.

8. Chacín-Bonilla L, Dikdan Y, Guanipa N, Villalobos R. Prevalencia de Entamoeba histolytica y otros parásitos intestinales en un barrio del municipio Mara, estado Zulia, Venezuela. Invest Clin 1990; 31: 3-15.

9. Chacín-Bonilla L. Cyclospora cayetanensis. In: Rose JB, Jiménez-Cisneros B, Eds. Global Water Pathogens Project. Lansing, MI: E. Lansing, UNESCO; 2017. P 1-43. https://www.waterpathogens.org/book/cyclospora-cayetanensis.

10. Harris NL, Loke P. Recent advances in type-2- cell- mediated immunity: insights from helminth infection. Immunity 2017; 47:1024-1036. 